hep-th/9906248

YITP-99-40

EFI-99-30

USC-99/HEP-M4

UT-KOMABA/99-10

\title{
ON THE QUANTIZATION OF NAMBU BRACKETS
}

\author{
Hidetoshi Awata ${ }^{a 1}$, Miao $\mathbf{L i}^{b 2}$ \\ and \\ Djordje Minic $^{c 3}$, Tamiaki Yoneya ${ }^{d 4}$ \\ ${ }^{a}$ Yukawa Institute for Theoretical Physics, Kyoto University, 606 Kyoto, \\ Japan \\ ${ }^{b}$ Enrico Fermi Institute, University of Chicago, 5640 Ellis Avenue, Chicago, \\ IL 60637, USA \\ ${ }^{c}$ Department of Physics and Astronomy, University of Southern California, \\ Los Angeles, CA 90089-0484, USA \\ ${ }^{d}$ Institute of Physics, University of Tokyo, Komaba, Meguro-ku, 153 Tokyo, \\ Japan
}

\begin{abstract}
We present several non-trivial examples of the three-dimensional quantum Nambu bracket which involve square matrices or three-index objects. Our examples satisfy two fundamental properties of the classical Nambu bracket: they are skew-symmetric and they obey the Fundamental Identity. We contrast our approach to the existing literature on the quantum deformations of Nambu mechanics. We also discuss possible applications of our results in M-theory.
\end{abstract}

\footnotetext{
${ }^{1}$ e-mail: awata@yukawa.kyoto-u.ac.jp

2e-mail: mli@theory.uchicago.edu

3 e-mail: minic@physics.usc.edu

${ }^{4}$ e-mail: tam@hep1.c.u-tokyo.ac.jp
} 


\section{Introduction}

In $1973 \mathrm{Nambu}$ [1] proposed a generalization of Hamiltonian mechanics and statistical mechanics involving odd-dimensional "phase-spaces". In particular, in the case of a three-dimensional "phase-space" labeled by $x, y, z$ Nambu proposed the following generalized Hamilton evolution equation

$$
\frac{d F}{d t}=\frac{\partial\left(H_{1}, H_{2}, F\right)}{\partial(x, y, z)}
$$

where the right hand side (R.H.S.) denotes the three-dimensional Jacobian of $H_{1}(x, y, z), H_{2}(x, y, z), F(x, y, z)$ with respect to $x, y, z$ and defines the classical Nambu bracket. Nambu's proposal was motivated by the general validity of the Liouville theorem, as it is apparent from the form of the evolution equation (1).

It was then noticed that various physical systems described by this formalism in $n$ "phase-space" dimensions can be realized as singular Hamiltonian systems in $2 n$ "phase-space" dimensions [2]. The original proposal of Nambu has also been given an elegant geometric formulation by Takhtajan [3].

The quantization problem of the classical Nambu bracket turned out to be very difficult. The only existing approach uses an unusual version of deformation quantization called Zariski quantization [4], [5]. However, an explicit realization of the quantum Nambu bracket in terms of matrices, as posed in the original paper by Nambu, still seems to be lacking.

It is one of the aims of this article to present several non-trivial examples of the quantum version of the Nambu bracket in terms of ordinary matrices and also in terms of three-index objects (cubic matrices). Our main motivation for this investigation comes from an expectation that the quantum Nambu bracket might turn out to be a useful technical tool in M-theory.

The article is organized as follows: first, in section 2, we briefly review the connection between volume preserving diffeomorphisms and the classical Nambu bracket, and point out some novel structural features of the latter. Then in section 3, we give an explicit matrix realization of the threedimensional quantum Nambu bracket which satisfies two crucial properties of its classical counterpart: skew-symmetry and the Fundamental Identity. We contrast our results to the existing examples obtained in the formalism of Zariski quantization [5]. In section 4, we give an explicit realization of the three-dimensional quantum Nambu bracket in terms of three-index objects, 
which we call cubic matrices. In section 5, we present the generalization of our approach to the $n$-dimensional quantum Nambu bracket. Finally in section 6, we discuss possible applications of our results in M-theory.

\section{Volume Preserving Diffeomorphisms and the Classical Nambu Bracket}

Consider a three-dimensional space parametrized by $\left\{x^{i}\right\}$. The three-dimensional volume preserving diffeomorphisms (VPD) on this space are described by a differentiable map

$$
x^{i} \rightarrow y^{i}(x)
$$

such that

$$
\left\{y^{1}, y^{2}, y^{3}\right\}=1
$$

where, by definition

$$
\{A, B, C\} \equiv \epsilon^{i j k} \partial_{i} A \partial_{j} B \partial_{k} C
$$

is the Nambu-Poisson (NP) bracket, or Nambu bracket, or Nambu triple bracket, which satisfies [3], [4], [5], [6]

1. Skew-symmetry

$$
\left\{A_{1}, A_{2}, A_{3}\right\}=(-1)^{\epsilon(p)}\left\{A_{p(1)}, A_{p(2)}, A_{p(3)}\right\},
$$

where $p(i)$ is the permutation of indices and $\epsilon(p)$ is the parity of the permutation,

2. Derivation

$$
\left\{A_{1} A_{2}, A_{3}, A_{4}\right\}=A_{1}\left\{A_{2}, A_{3}, A_{4}\right\}+\left\{A_{1}, A_{3}, A_{4}\right\} A_{2},
$$

3. Fundamental Identity (FI-1) [3], [6]

$$
\begin{array}{r} 
\\
\left\{\left\{A_{1}, A_{2}, A_{3}\right\}, A_{4}, A_{5}\right\}+\left\{A_{3},\left\{A_{1}, A_{2}, A_{4}\right\}, A_{5}\right\} \\
+\left\{A_{3}, A_{4},\left\{A_{1}, A_{2}, A_{5}\right\}\right\}=\left\{A_{1}, A_{2},\left\{A_{3}, A_{4}, A_{5}\right\}\right\} .
\end{array}
$$


The three-dimensional VPD involves two independent functions. Let these functions be denoted by $f$ and $g$. The infinitesimal three-dimensional VPD generator is then given as

$$
\begin{aligned}
D(f, g) & \equiv \epsilon^{i j k} \partial_{i} f \partial_{j} g \partial_{k} \\
& \equiv D^{k}(f, g) \partial_{k} .
\end{aligned}
$$

The volume-preserving property is nothing but the identity

$$
\partial_{i} D^{i}(f, g)=\partial_{k}\left(\epsilon^{i j k} \partial_{i} f \partial_{j} g\right)=0 .
$$

Given an arbitrary scalar function $X\left(x^{i}\right)$, the three-dimensional VPD acts as

$$
D(f, g) X=\{f, g, X\} .
$$

Apart from the issue of global definition of the functions $f$ and $g$, we can represent an arbitrary infinitesimal volume-preserving diffeomorphism in this form.

On the other hand, if the base three-dimensional space $\left\{x^{i}\right\}$ is mapped into a target space of dimension $d+1$ whose coordinates are $X^{\alpha}(\alpha=$ $0,1,2, \ldots, d)$, the induced infinitesimal volume element is

$$
d \sigma \equiv \sqrt{\left\{X^{\alpha}, X^{\beta}, X^{\gamma}\right\}^{2}} d x^{1} d x^{2} d x^{3},
$$

provided the target space is a flat Euclidean space. The volume element is of course invariant under the general three-dimensional diffeomorphisms.

The triple product $\left\{X^{\alpha}, X^{\beta}, X^{\gamma}\right\}$ is also "invariant" under the VPD. Or more precisely, it transforms as a scalar. Namely,

$$
\left\{Y^{\alpha}, Y^{\beta}, Y^{\gamma}\right\}-\left\{X^{\alpha}, X^{\beta}, X^{\gamma}\right\}=\epsilon D(f, g)\left\{X^{\alpha}, X^{\beta}, X^{\gamma}\right\}+O\left(\epsilon^{2}\right)
$$

for

$$
Y=X+\epsilon D(f, g) X .
$$

This is due to the the Fundamental Identity FI-1 which shows that the operator $D(f, g)$ acts as a derivation within the NP bracket. For fixed $f$ and $g$, we can define a finite transformation by

$$
X(t) \equiv \exp (t D(f, g)) \rightarrow X=\sum_{n=0}^{\infty} \frac{t^{n}}{n !}\{f, g,\{f, g,\{\ldots,\{f, g,\{f, g, X\}\} \ldots,\}\}\}
$$


which satisfies the Nambu "equation of motion" [1]

$$
\frac{d}{d t} X(t)=\{f, g, X(t)\}
$$

The Nambu-Poisson structure is preserved under this evolution equation.

Let us now consider the group property of the three-dimensional VPD. First we derive, using the FI-1

$$
\begin{aligned}
{\left[D\left(f_{1}, g_{1}\right), D\left(f_{2}, g_{2}\right)\right] X } & \equiv\left\{f_{1}, g_{1},\left\{f_{2}, g_{2}, X\right\}\right\}-\left\{f_{2}, g_{2},\left\{f_{1}, g_{1}, X\right\}\right\} \\
& =\left\{\left\{f_{1}, g_{1}, f_{2}\right\}, g_{2}, X\right\}+\left\{f_{2},\left\{f_{1}, g_{1}, g_{2}\right\}, X\right\} .
\end{aligned}
$$

The second line, however, does not have manifest antisymmetry under the interchange $1 \leftrightarrow 2$. To understand what this means, we note that the Nambu bracket defined by (4) satisfies the following identities in addition to the FI-1 as pointed out by Hoppe [7]

1. FI-2

$$
\left\{A_{[1}, A_{2},\left\{A_{3}, A_{4]}, B\right\}\right\}=0
$$

2. FI-3

$$
\begin{aligned}
& \left\{\left\{A_{1}, A_{2}, A_{3}\right\}, A_{4}, B\right\}-\left\{\left\{A_{2}, A_{3}, A_{4}\right\}, A_{1}, B\right\} \\
& +\left\{\left\{A_{3}, A_{4}, A_{1}\right\}, A_{2}, B\right\}-\left\{\left\{A_{4}, A_{1}, A_{2}\right\}, A_{3}, B\right\}=0 .
\end{aligned}
$$

Here we changed the order of the elements, using skew-symmetry, from Hoppe's original form. It is claimed in [7] that two of the identities FI-1, FI-2, FI-3 are independent. We wish to show that FI-2 and FI-3 can be derived from FI-1 and skew-symmetry.

The identity FI-2 and skew-symmetry property enable us to rewrite (17) as

$$
\begin{gathered}
\left\{\left\{f_{1}, g_{1}, f_{2}\right\}, g_{2}, X\right\}+\left\{f_{2},\left\{f_{1}, g_{1}, g_{2}\right\}, X\right\}=-\left\{\left\{f_{2}, g_{2}, f_{1}\right\}, g_{1}, X\right\} \\
-\left\{f_{1},\left\{f_{2}, g_{2}, g_{1}\right\}, X\right\} .
\end{gathered}
$$

Thus we recover the required antisymmetry under $1 \leftrightarrow 2$.

However, in our case, the left hand side (L.H.S.) of the first line in (17) is antisymmetric by definition, and therefore (20) must hold identically. Thus 
we conclude that the identity (20) is actually a consequence of the original fundamental identity FI-1, contrary to Hoppe's statement [7].

Note that in going from the first line to the second line in (17), the explicit form of the Nambu bracket need not be used, nor the property of skew-symmetry. If the property of skew-symmetry with respect to the first two entries is assumed, (20) is equivalent to the FI-3. Hence, the FI-2 is also a consequence of the FI-1.

The algebra of $D(f, g)$ now takes the form

$$
\begin{aligned}
{\left[D\left(f_{1}, g_{1}\right), D\left(f_{2}, g_{2}\right)\right] } & =\frac{1}{2}\left(D\left(\left\{\left\{f_{1}, g_{1}\right\}, g_{1}\right\}, g_{2}\right)+D\left(f_{2},\left\{f_{1}, g_{1}, g_{2}\right\}\right)\right. \\
& \left.-D\left(\left\{f_{2}, g_{2}, f_{1}\right\}, g_{1}\right)-D\left(f_{1},\left\{f_{2}, g_{2}, g_{1}\right\}\right)\right) .
\end{aligned}
$$

One important lesson is that the skew-symmetry property (5) is not necessary for the group structure of the transformations generated by $D(f, g)$. What is then the role of skew-symmetry from the view point of symmetry? One obvious fact is that the property of skew-symmetry with respect to the first and the second entries means that only the "independent" part of the two parameter functions $f$ and $g$ contributes to the transformation, in the sense that

$$
D(f, g)=D(f+c g, g)=D(f, g+c f)
$$

for arbitrary constant $c$.

In the case of the usual Poisson structure, the algebra of two-dimensional area preserving diffeomorphisms is given by

$$
\left[D\left(f_{1}\right), D\left(f_{2}\right)\right]=D\left(f_{3}\right)
$$

where

$$
\begin{gathered}
f_{3}=\left\{f_{1}, f_{2}\right\} \\
D(f) X=\{f, X\}
\end{gathered}
$$

Formally, the three-dimensional VPD algebra should also be expressible in the form

$$
\left[D\left(f_{1}, g_{1}\right), D\left(f_{2}, g_{2}\right)\right]=D\left(f_{3}, g_{3}\right)
$$

Is it indeed possible to express $\left(f_{3}, g_{3}\right)$ in terms of the Nambu bracket? It turns out that the three-dimensional analogue of the commutator algebra

$$
D\left(A_{[1}\right) D\left(A_{2]}\right)=D\left(\left\{A_{1}, A_{2}\right\}\right)
$$


can be written using the quantum triple Nambu commutator [1]

$$
[A, B, C]_{N} \equiv A B C-A C B+B C A-B A C+C A B-C B A
$$

as follows

$$
D\left(A_{[1}, A_{2}\right) D\left(A_{3]_{N}}, B\right)=2 D\left(\left\{A_{1}, A_{2}, A_{3}\right\}, B\right),
$$

or equivalently

$$
D\left(B_{[1}, B_{2}\right) D\left(A_{[1}, A_{2}\right) D\left(A_{3]_{N}}, B_{3]_{N}}\right)=4 D\left(\left\{A_{1}, A_{2}, A_{3}\right\},\left\{B_{1}, B_{2}, B_{3}\right\}\right) .
$$

Both relations are equivalent to the Fundamental Identity

$$
\begin{aligned}
\left\{A_{1}, A_{2},\left\{A_{3}, B, C\right\}\right\}+ & \left\{A_{2}, A_{3},\left\{A_{1}, B, C\right\}\right\}+\left\{A_{3}, A_{1},\left\{A_{2}, B, C\right\}\right\} \\
& =\left\{\left\{A_{1}, A_{2}, A_{3}\right\}, B, C\right\}
\end{aligned}
$$

This result suggests that there is a new kind of symmetry based on a new composition law whose infinitesimal algebra is given by the triple commutator (28). As far as we know this type of symmetry has not been observed previously in the literature. It is tempting to conjecture that this symmetry is related to the gauge transformations that are not of Yang-Mills type as in [8].

\section{Square Matrices and the Quantum Nambu Brackets}

The problem of discretization of p-dimensional volume preserving diffeomorphisms is related to the issue of quantization of the p-dimensional Nambu bracket. Apparently there exists in the literature only one solution of the quantization problem of Nambu brackets which is based on a non-standard deformation quantization called Zariski quantization [4]. The quantum Nambu bracket constructed in [4] is skew-symmetric and obeys both the derivation property and the Fundamental Identity. There also exists an example of a quantum Nambu bracket constructed via the same deformation quantization procedure, which is skew-symmetric and obeys the Fundamental Identity, but does not satisfy the derivation property [5].

In this section we wish to give an explicit matrix realization of the quantum Nambu bracket, which is skew-symmetric and obeys the Fundamental 
Identity. Our simple example should be contrasted to the example constructed in [5].

What do we mean by a quantum triple Nambu bracket? In general we want an object $[F, G, H]$ which satisfies properties analogous to the classical Nambu bracket $\{f, g, h\}$ as listed in the previous section. (Here $f, g, h$ are functions of three variables, and the nature of $F, G, H$ is left open for the moment.) Thus $[F, G, H]$ is expected to satisfy [3], [4], [5], [6]

1. Skew-symmetry

$$
\left[A_{1}, A_{2}, A_{3}\right]=(-1)^{\epsilon(p)}\left[A_{p(1)}, A_{p(2)}, A_{p(3)}\right],
$$

where again $p(i)$ is the permutation of indices and $\epsilon(p)$ is the parity of the permutation,

2. Derivation

$$
\left[A_{1} A_{2}, A_{3}, A_{4}\right]=A_{1}\left[A_{2}, A_{3}, A_{4}\right]+\left[A_{1}, A_{3}, A_{4}\right] A_{2},
$$

3. Fundamental Identity (F.I.)

$$
\begin{array}{r}
{\left[\left[A_{1}, A_{2}, A_{3}\right], A_{4}, A_{5}\right]+\left[A_{3},\left[A_{1}, A_{2}, A_{4}\right], A_{5}\right]} \\
+\left[A_{3}, A_{4},\left[A_{1}, A_{2}, A_{5}\right]\right]=\left[A_{1}, A_{2},\left[A_{3}, A_{4}, A_{5}\right]\right] .
\end{array}
$$

(Note that the two-dimensional quantum Nambu bracket which satisfies above properties is just the usual commutator of matrices $[A, B] \equiv A B-B C$. In this case the F.I. reduces to the Jacobi identity.)

First we point out that there exists a matrix realization of the triple quantum Nambu bracket which satisfies the property of skew-symmetry and the Fundamental Identity. To demonstrate this claim we define a totally antisymmetric triple bracket of three matrices A, B, C as

$$
[A, B, C] \equiv(\operatorname{tr} A)[B, C]+(\operatorname{tr} B)[C, A]+(\operatorname{tr} C)[A, B]
$$

Then $\operatorname{tr}[A, B, C]=0$, and if $C=1,[A, B, 1]=N[A, B]$, where $N$ is the rank of square matrices.

It is quite easy to prove the Fundamental Identity now. The F.I. is just an adjoint action involving matrices $X, Y, A, B, C$

$$
[X, Y,[A, B, C]]=[[X, Y, A], B, C]+[A,[X, Y, B], C]+[A, B,[X, Y, C]] .
$$


Using the definition of the triple bracket, the L.H.S. is equal to

$$
\begin{gathered}
(\operatorname{tr} A)[X, Y,[B, C]]+c(A, B, C)=\operatorname{tr} A \operatorname{tr} X[Y,[B, C]]-\operatorname{tr} A \operatorname{tr} Y[X,[B, C]] \\
+c(A, B, C)
\end{gathered}
$$

where $c(A, B, C)$ denotes the cyclic permutation of $A, B, C$.

On the other hand, the R.H.S. of the F.I. is equal to

$$
(\operatorname{tr} X)[[Y, A], B, C]+(\operatorname{tr} Y)[[A, X], B, C]+(\operatorname{tr} A)[[X, Y], B, C]+c(A, B, C)
$$

It is easy to see that

$$
(\operatorname{tr} A)[[X, Y], B, C]+(\operatorname{tr} B)[[X, Y,], C, A]+(\operatorname{tr} C)[[X, Y], A, B]=0 .
$$

This is because each term is proportional to a product of two traces of the three matrices $A, B, C$, and they cancel. For instance, from the first term in the above equation we have $(\operatorname{tr} A \operatorname{tr} B)[C,[X, Y]]$, while from the second term we have $(\operatorname{tr} B \operatorname{tr} A)[[X, Y], C]$ - the two add up to zero. Because of the above equation, the R.H.S. of the F.I. simplifies to $(\operatorname{tr} X)[[Y, A], B, C]+$ $(\operatorname{tr} Y)[[A, X], B, C]+c(A, B, C)$.

We can now see why the L.H.S. is equal to the R.H.S. From both the L.H.S. and R.H.S. every term upon expansion is proportional to a product of two traces, one comes from $\operatorname{tr} X$ or $\operatorname{tr} Y$, another from $\operatorname{tr} A, \operatorname{tr} B$ or $\operatorname{tr} C$. For example, on the L.H.S. there is a term

$$
(\operatorname{tr} A \operatorname{tr} X)[Y,[B, C]]
$$

while from the R.H.S., we find

$$
(\operatorname{tr} A \operatorname{tr} X)([B,[Y, C]]-[C,[Y, B]])
$$

This term is equal to the one from the L.H.S. by virtue of the Jacobi identity. The same is true for other terms, simply by doing permutations. This completes the proof of the Fundamental Identity.

Given our example of a three-dimensional quantum Nambu bracket, let us consider the following "gauge transformation"

$$
\delta A \equiv i[X, Y, A],
$$


where we introduce the factor $i$, because we take all matrices to be Hermitian. This transformation represents an obvious quantum form of the three-dimensional volume preserving diffeomorphisms.

By the definition of the triple bracket, the gauge transformation takes the following explicit form

$$
\delta A=i([(\operatorname{tr} X) Y-(\operatorname{tr} Y) X, A]+(\operatorname{tr} A)[X, Y])
$$

where the first term in the parentheses is just the usual $s u(N)$ gauge transformation, and the second term is apparently new.

The first important property of the gauge transformation, in addition to satisfying the generalized composition rule (or the F.I.), is that $\delta \operatorname{tr}(A B)=0$ provided $\operatorname{tr} A=\operatorname{tr} B=0$. To see this look at

$$
\delta \operatorname{tr}(A B)=i[\operatorname{tr}([X, Y, A] B)+\operatorname{tr}([X, Y, B] A)]
$$

which in turn is equal to

$$
i[\operatorname{tr}([(\operatorname{tr} X) Y-(\operatorname{tr} Y) X, A] B)+(A \leftrightarrow B)]=0 .
$$

The crucial point is that $\operatorname{since} \operatorname{tr} A=0$, the last term in the gauge transformation (40) is absent, therefore the gauge transformation is just an $s u(N)$ gauge transformation. Thus, we have the following general result:

If $\operatorname{tr} A_{i}=0, i=1, \ldots n$, then

$$
\operatorname{tr}\left(A_{1} A_{2} \ldots A_{n}\right)
$$

is gauge invariant.

Note that the gauge transformation of a commutator does not satisfy the usual composition rule, namely

$$
[X, Y,[A, B]] \neq[[X, Y, A], B]+[A,[X, Y, B]] .
$$

To see this, we only need to realize that the gauge transformation of $[A, B]$ is just an $s u(N)$ transformation, since $\operatorname{tr}[A, B]=0$. On the other hand, there is an additional term on the R.H.S. of the above inequality, $(\operatorname{tr} A)[[X, Y], B]+$ $(\operatorname{tr} B)[A,[X, Y]]$. If $\operatorname{tr} A=\operatorname{tr} B=0$, then the gauge transformation of the commutator $[A, B]$ satisfies the composition rule. Similar comments apply to $\left[\left[X^{i}, X^{j}\right],\left[X^{l}, X^{k}\right]\right]$, since although $\operatorname{tr}\left[X^{i}, X^{j}\right]=0,\left[X^{i}, X^{j}\right]$ itself does not satisfy the composition rule. 
We can also define a triple quantum Nambu bracket involving fermionic matrices. If there is only one fermionic matrix involved in a triple bracket, the definition is the same as in (35), and the F.I. identity holds. If there are two fermionic matrices, $\psi$ and $\lambda$, define

$$
[A, \psi, \lambda]=(\operatorname{tr} A)\{\psi, \lambda\}+(\operatorname{tr} \psi)[\lambda, A]+(\operatorname{tr} \lambda)[\psi, A],
$$

where $\{\psi, \lambda\}=\psi \lambda+\lambda \psi$, and $\operatorname{tr}\{\psi, \lambda\}=0$. Due to this property and the Jacobi identity involving fermionic matrices, the F.I. still holds. Note that this triple bracket is symmetric in $\psi$ and $\lambda$.

If all three matrices are fermionic, we can define the following fermionic triple quantum Nambu bracket

$$
[\psi, \lambda, \chi]=(\operatorname{tr} \psi)\{\lambda, \chi\}+(\operatorname{tr} \lambda)\{\chi, \psi\}+(\operatorname{tr} \chi)\{\psi, \lambda\} .
$$

Obviously this triple bracket is totally symmetric in three fermionic matrices. Again we can repeat the same steps as before to show that the F.I. holds.

Notice that the form of the gauge transformation (40) indicates that a bosonic Hermitian matrix $A$ can be transformed into a form proportional to the unit $N \times N$ matrix as long as $\operatorname{tr} A \neq 0$. In other words, since the gauge transformation is traceless, one can show that a Hermitian matrix can be brought to the following form

$$
A \rightarrow \frac{1}{N} \operatorname{tr} A 1_{N} .
$$

To prove this note that the first term in (40) represents the usual $s u(N)$ gauge transformation, and it can be used to diagonalize $A$; the second term helps to balance all the eigenvalues of $A$. Hence, to diagonalize $A$ first, choose $X$ to be a Hermitian matrix and $Y=1$, so that $\delta A=i N[A, X]-$ which is a standard $s u(N)$ gauge transformation. Once $A$ is diagonalized this way, choose $X, Y \in s u(N)$, so that the first term in (40) is absent, and $\delta A=i(\operatorname{tr} A)[X, Y]$. By choosing suitable $X$ and $Y$, we can let $[X, Y]$ be any element in the Cartan algebra of $s u(N)$, so that any residual of $A$ in this Cartan algebra can be gauged away, thus proving the above statement.

One final remark: one might wonder whether there exists a "c"-number for our three-dimensional quantum Nambu bracket (35), i.e. whether there exists an element $E$ such that

$$
[E, A, B]=0
$$


for arbitrary elements $A, B$ ? It appears that there is no solution for this condition except $E=0$ for the triple Nambu bracket defined above.

Notice also that if we consider the triple commutator of the form

$$
[G, A, B]
$$

it follows from the property of skew-symmetry that this bracket is invariant under $A \rightarrow A+G$ and $B \rightarrow B+G$. In other words, $[G, G, B]=[G, A, G]=0$ precisely because of the property of skew-symmetry.

\section{Cubic Matrices and the Nambu Bracket}

In this section we want to address the following question: Is there a manyindex matrix representation of the three-dimensional quantum Nambu bracket? For example, the three-dimensional classical Nambu bracket is naturally realized in terms of functions of three variables. Then it is natural to ask: Is it possible to realize the three-dimensional quantum Nambu bracket in terms of three-index objects $A_{i j k}$ (cubic matrices)? It turns out that the answer to this question is positive. In this section we give some explicit examples of the three-dimensional quantum Nambu bracket written in terms of three-index objects or cubic matrices.

Let us introduce the following generalization of the traces

$$
\langle A\rangle \equiv \sum_{p m} A_{p m p}, \quad\langle A B\rangle \equiv \sum_{p q m} A_{p m q} B_{q m p}, \quad\langle A B C\rangle \equiv \sum_{p q r m} A_{p m q} B_{q m r} C_{r m p}
$$

which satisfy $\langle A B\rangle=\langle B A\rangle$ and $\langle A B C\rangle=\langle B C A\rangle=\langle C A B\rangle$. Let us furthermore define a triple-product

$$
(A B C)_{i j k} \equiv \sum_{p} A_{i j p}\langle B\rangle C_{p j k}=\sum_{p q m} A_{i j p} B_{q m q} C_{p j k}
$$

Given this triple-product we define the following skew-symmetric quantum Nambu bracket

$$
[A, B, C] \equiv(A B C)+(B C A)+(C A B)-(C B A)-(A C B)-(B A C) .
$$

The middle index $j$ of $A_{i j k}$ can be treated as an internal index for the matrix

realization of the three-dimensional quantum Nambu bracket we considered 
in the previous section. Therefore we expect that the F.I. should be satisfied. This indeed turns out to be the case. Note also that $\langle(A B C)\rangle=\langle B\rangle\langle A C\rangle \neq$ $\langle A B C\rangle$ and $\langle(A B C) D\rangle=\langle B\rangle\langle A C D\rangle$.

Then by using the following relations

$$
\begin{aligned}
& ((A B C) D E)=((A D C) B E)=(A B(C D E))=(A D(C B E)), \\
& (A(B C D) E)=(A(D C B) E),
\end{aligned}
$$

one can directly prove that the skew-symmetric Nambu bracket (47) with the triple-product (46) obeys the F.I. (34)

The "trace" $\langle A B\rangle$ has the property

$$
\langle[X, Y, A] B\rangle+\langle A[X, Y, B]\rangle=0,
$$

provided $\langle A\rangle=\langle B\rangle=0$. Therefore, since $\langle[A, B, C]\rangle=0$ for any threeindex objects $A, B$ and $C$, the trace of the product of Nambu brackets $\langle[A, B, C][D, E, F]\rangle$ is gauge invariant. Notice that if we generalize the trace $(45)$ as

$$
\left\langle A^{1} A^{2} \cdots A^{n}\right\rangle \equiv \sum_{p_{1}, p_{2}, \cdots, m} A_{p_{1} m p_{2}}^{1} A_{p_{2} m p_{3}}^{2} \cdots A_{p_{n} m p_{1}}^{n}, \quad n=1,2, \cdots,
$$

we can also demonstrate that the trace of any product of Nambu brackets $\langle[A, B, C][D, E, F] \cdots[X, Y, Z]\rangle$ is gauge invariant.

Let us also define $I_{i j k} \equiv \delta_{i k}^{(j)}$, where $\delta_{i k}^{(j)}=0$, if $i \neq k$, for any $j$ and $\delta_{i k}^{(j)}=1$, if $i=k$, for any $j$. Then

$$
\begin{array}{cc}
(A I B)=\langle I\rangle \sum_{p} A_{i j p} B_{p j k}, & (I A B)=(B A I)=\langle A\rangle B, \\
(I A I)=\langle A\rangle \delta_{i k}^{(j)}, & (I I A)=(A I I)=\langle I\rangle A,
\end{array}
$$

and $[A, I, B]=\sum_{p}\left(A_{i j p} B_{p j k}-B_{i j p} A_{p j k}\right)$. Hence for any middle index $j$, $[A, I, B]$ reduces to the usual commutator $\left[A^{(j)}, B^{(j)}\right]$ for the matrices $A_{i k}^{(j)} \equiv$ $A_{i j k}$ and $B_{i k}^{(j)} \equiv B_{i j k}$.

Finally we list other examples of triple-products $(A B C)_{i j k}$ which also satisfy the same relations as eq. (48) and hence lead to the F.I. for the skewsymmetric Nambu bracket (47)

$$
\sum_{p q} A_{i j p} B_{q j q} C_{p j k}, \quad \sum_{p q m n} A_{i j p} B_{q m q} C_{p n k}, \quad \sum_{p q m n} A_{i n p} B_{q m q} C_{p j k} .
$$




\section{Generalization: n-dimensional Quantum Nambu Bracket}

We can now generalize and formalize our construction for the $n$-dimensional quantum Nambu bracket which obeys the $n$-dimensional Fundamental Identity $F I_{n}$ :

$$
\left[X_{1}, \cdots, X_{n-1},\left[A_{1}, \cdots, A_{n}\right]\right]=\sum_{i=1}^{n}\left[A_{1}, \cdots,\left[X_{1}, \cdots, X_{n-1}, A_{i}\right], \cdots, A_{n}\right]
$$

Let $A_{i}$ be operators and let $\left\langle A_{i}\right\rangle$ be some $c$-numbers ("traces"). Let us define a generalized $n$-dimensional bracket given an $(n-1)$-dimensional bracket as follows:

$$
\left[A_{1}, A_{2}, \cdots, A_{n}\right] \equiv \sum_{i=1}^{n}(-1)^{i-1}\left\langle A_{i}\right\rangle\left[A_{1}, \cdots, \check{A}_{i}, \cdots, A_{n}\right] .
$$

Here $\check{A}_{i}$ stands for the term that is omitted. Moreover, let us assume that the "trace" $\langle A\rangle$ has the property $\left\langle\left[A_{1}, \cdots, A_{n}\right]\right\rangle=0$. Note that if the $(n-1)$ dimensional bracket is also related to the $(n-2)$-dimensional bracket with the same "trace" $\langle A\rangle$ as in the $n$-dimensional case, then $\left[A_{1}, \cdots, A_{n}\right]=0$.

By a straightforward computation, one can show that if an $(n-1)$ dimensional Nambu bracket is skew-symmetric and satisfies $F I_{n-1}$ then the corresponding $n$-dimensional bracket is also skew-symmetric and obeys $F I_{n}$ (52).

\section{Conclusions}

In this article we have constructed several explicit examples of the quantum Nambu bracket in terms of square matrices and three-index objects - cubic matrices. Our examples satisfy two crucial properties of the classical Nambu bracket: skew-symmetry and the Fundamental Identity. Our results should be compared to the existing literature on the deformation quantization approach to Nambu mechanics [5]. We have also discussed the generalization

of our approach to the $n$-dimensional quantum Nambu bracket. Notice that it still remains on open question whether all three properties of the classical 
Nambu bracket - skew-symmetry, derivation and the F.I. - can be realized in terms of square or cubic matrices.

We wish to conclude this article by outlining a few possible applications of our formulation. One possible application of our results is in relation to the yet unknown mathematical structure of the space-time uncertainty relation in M-theory [9]. There seems to exists a naive similarity between the form of the space-time uncertainty relation in M-theory and the threedimensional quantum Nambu bracket [9]. It would be interesting to formulate this intuitive relation more precisely. Also, in view of the fact that spacetime uncertainty principle captures important qualitative features of Matrix theory [10] it would be very interesting to see whether our results could be relevant for the still unsolved problem of covariantization of Matrix theory [11]. One universal technical aspect of any attempt to covariantize Matrix theory is to promote all spacetime coordinates to matrices, and then to gauge fix a matrix, say the light-cone time matrix, to proportional to the identity matrix. This demands enlarging the gauge symmetry. Our model of Nambu bracket presented in sect. 3 gives rise to gauge symmetry large enough to achieve this goal.

Furthermore, given the fact that the light-cone action for a super p-brane is invariant under the volume preserving diffeomorphisms [8] and given the obvious relationship between $\mathrm{p}$-dimensional $(p \geq 2)$ volume preserving diffeomorphisms and p-dimensional classical Nambu brackets [12], [13], [8], we expect that our results should shed some light on the quantization problem of p-branes in M-theory and the unknown structure of the non-Abelian antisymmetric tensor gauge theory that emerges in the case when $p \geq 3$ [8].

We hope to return to some of these problems in the future.

\section{Acknowledgements}

It is our pleasure to thank T. Banks, I. Bars, O. Bergman, S. Chaudhuri, E. Gimon, M. Günaydin, P. Horava, T. Hübsch, J. Minahan, Y. Nambu, J. Polchinski, K. Pilch, J. Schwarz, L. Smolin, P. Wiegmann, E. Witten and C. Zachos for interesting comments and discussions. D. M. would like to thank Tokyo University and the Yukawa Institute for Theoretical Physics for their hospitality during the final stage of this work. The work of M.L. is supported in part by DOE grant DE-FG02-90ER-40560 and NSF grant PHY 91-23780. The work of D. M. is supported in part by DOE grant DE-FG03-84ER40168 
and by a National Science Foundation grant NSF9724831 for collaborative research between USC and Japan. The work of T.Y. is supported in part by Grant-in-Aid for Scientific Research (No. 09640337) and Grant-in-Aid for International Scientific Research (Joint Research, N0. 10044061) from the Ministry of Education, Science and Culture.

\section{Appendix}

Sufficient Condition for the F.I.

In this appendix we will give a sufficient condition for the F.I. (34). To find this, it is worth to recall the classical case: Why does the Nambu Poisson bracket obey the classical F.I.?

For the usual Poisson bracket $\{A, B\}=A_{p} B_{q}-B_{p} A_{q}$ with $A_{p} \equiv \partial_{p} A$, it is natural to define a non-commutative product as $(A B) \equiv A_{p} B_{q}$. Then

$$
\begin{aligned}
& ((A B) C)=A_{p} B_{p q} C_{q}+A_{p p} B_{q} C_{q} \\
& (A(B C))=A_{p} B_{p} C_{q q}+A_{p} B_{p q} C_{q}
\end{aligned}
$$

We thus distinguish three kinds of triple-products: $A_{p} B_{p q} C_{q}, A_{p p} B_{q} C_{q}$ and $A_{p} B_{p} C_{q q}$. The second triple-product is symmetric under the interchange of $B_{q}$ and $C_{q}$; the third is symmetric under the interchange of $A_{p}$ and $B_{p}$.

We can generalize this to the quantum case. Let us define three kinds of triple-products $(A B C)^{M} M=0,23,12$ which possess symmetries of their classical counterparts $(A B C)^{23}=(A C B)^{23}$ and $(A B C)^{12}=(B A C)^{12}$. Then a sufficient condition for the Jacobi identity is

$$
\begin{aligned}
& ((A B) C)=(A B C)^{0}+(A B C)^{23} \\
& (A(B C))=(A B C)^{12}+(A B C)^{0} .
\end{aligned}
$$

For the usual square matrices, only one triple-product, $(A B C)^{0}$, is non-zero and hence the sufficient condition is nothing but the property of associativity $((A B) C)=(A(B C))$, as expected.

In the case of a three-dimensional Nambu bracket, the associativity is too weak for the F.I., and the corresponding sufficient condition turns out to be more complicated. If we define the classical Nambu triple-product as $(A B C) \equiv A_{p} B_{q} C_{r}$, in analogy with the treatment of the Poisson bracket, 
then

$$
\begin{aligned}
& ((A B C) D E)=A_{p} B_{q} C_{p r} D_{q} E_{r}+A_{p} B_{p q} C_{r} D_{q} E_{r}+A_{p p} B_{q} C_{r} D_{q} E_{r}, \\
& (A(B C D) E)=A_{p} B_{p q} C_{q} D_{r} E_{r}+A_{p} B_{p} C_{q q} D_{r} E_{r}+A_{p} B_{p} C_{q} D_{q r} E_{r}, \\
& (A B(C D E))=A_{p} B_{q} C_{p} D_{q} E_{r r}+A_{p} B_{q} C_{p} D_{q r} E_{r}+A_{p} B_{q} C_{p r} D_{q} E_{r}(\mathrm{~A} .3)
\end{aligned}
$$

Thus we have to consider six kinds of quintuple-products which possess various obvious symmetries.

Again we can generalize this to the quantum case. Let us consider six kinds of quintuple-product with symmetries analogous to their classical counterparts

$$
\begin{aligned}
(A B C D E)^{24} & =(A D C B E)^{24} \\
(A B C D E)^{35} & =(A B E D C)^{35}, \\
(A B C D E)^{13} & =(C B A D E)^{13} \\
(A B C D E)^{24,35} & =(A D C B E)^{24,35}=(A B E D C)^{24,35} \\
(A B C D E)^{12,45} & =(B A C D E)^{12,45}=(A B C E D)^{12,45} \\
(A B C D E)^{13,24} & =(C B A D E)^{13,24}=(A D C B E)^{13,24} .
\end{aligned}
$$

Thus we have found that the sufficient condition for the F.I. for the skewsymmetric Nambu bracket (47) is

$$
\begin{aligned}
& ((A B C) D E)=(A B C D E)^{24}+(A B C D E)^{35}+(A B C D E)^{24,35} \\
& (A(B C D) E)=(A B D C E)^{35}+(A B C D E)^{12,45}+(A C B D E)^{13} \\
& (A B(C D E))=(A B C D E)^{13,24}+(A B C D E)^{13}+(A B C D E)^{24} .(\mathrm{A} .5)
\end{aligned}
$$

Note that the condition of associativity $((A B C) D E)=(A(B C D) E)=$ $(A B(C D E))$ does not guarantee the F.I. unlike the case of matrices, where the Jacobi identity is implied by the associativity of the matrix product. The examples in section 4 . are such that only one quintuple-product $(A B C D E)^{24}$ is non-zero and $(A(B C D) E)$ is a trivial quintuple-product which implies $(A[B C D] E)=0$.

\section{References}

[1] Y. Nambu, Phys. Rev. D7 (1973) 2405.

[2] F. Bayen and M. Flato, Phys. Rev. D11 (1975) 3049. 
[3] L. Takhtajan, Comm. Math. Phys. 160 (1994) 295.

[4] G. Dito, M. Flato, D. Sternheimer and L. Takhtajan, Comm. Math. Phys. 183 (1997) 1.

[5] G. Dito and M. Flato, Lett. Math. Phys. 39 (1997) 107.

[6] V. T. Filipov, Sib. Math. Jour. 26 no. 6 (1985) 126; M. Flato and C. Fronsdal, unpublished.

[7] J. Hoppe, Helv. Phys. Acta 70 (1997) 302.

[8] E. Bergshoeff, E. Sezgin, Y. Tanii and P. K. Townsend, Ann. Phys. 199 (1990) 340.

[9] T. Yoneya, Mod. Phys. Lett. A4 (1989) 1587. See also T. Yoneya, in "Wandering in the Fields". K. Kawarabayashi and A. Ukawa, eds. (World Scientific, 1987) pp.419; and T. Yoneya, in "Quantum String Theory", N. Kawamoto and T. Kugo, eds. (Springer, 1988) pp.23; T. Yoneya, Prog. Thor. Phys. 97 (1997) 949; M. Li and T. Yoneya, hepth/9611072, Phys. Rev. Lett. 78 (1997) 1219; M. Li and T. Yoneya, hep-th/9806240; D. Minic, Phys. Lett. B442 (1998) 102; C.S. Chu, P.M. Ho and Y.C. Kao, hep-th/9904133.

[10] T. Banks, W. Fischler, S. H. Shenker and L. Susskind, Phys. Rev. D55 (1997) 5112.

[11] H. Awata and D. Minic, JHEP04 (1998) 006; K. Fujikawa and K. Okuyama, Phys. Lett. B411 (1997) 261; L. Smolin, Phys. Rev. D57 (1998) 6216.

[12] E. Bergshoeff, E. Sezgin and P. K. Townsend, Phys. Lett. 189B (1987) 75; Ann. Phys. 185 (1988) 330; E. Bergshoeff, E. Sezgin and Y. Tanii, Nucl. Phys. B298 (1988) 187; B. de Wit, J. Hoppe and H. Nicolai, Nucl. Phys. B305 (1988) 545; I. Bars, Nucl. Phys. B343 (1990) 398.

[13] J. Goldstone, unpublished; J. Hoppe, MIT Ph.D. thesis, 1982 and in "Proc. Int. Workshop on Constraint's Theory and Relativistic Dynamics", G. Longhi and L. Lusanna, eds. (World Scientific, 1987); J. Hoppe, Int. J. Mod. Phys. A4 (1989) 5235; D. Fairlie, P. Fletcher and C. Zachos, J. Math. Phys. 31 (1990) 1088. 